From the Pediattic Service of the Mount Sinai Hospital.

New York.

\title{
ALIMENTARY TOXICOSIS
}

Por el Dr. SAMUEL KARELITZ, M. D. PH, B.

The subject of alimentary toxicosis has occupied pediatric thought continuously for several decades during which time great progress has been made. Renewed interest has been aroused in the United States by the appearance of epidemic diarrhea of the newborn and more recently by the better understanding of the intracellular electrolyte and water exchange. Datrow ${ }^{2}$, having shown a potassium deficit in children ill with severe diarrhea, has recommended the use of potassium in latger quantities than had bitherto been considered safe. With this addition to a system of therapy previously employed, he and Govan ${ }^{2}$ claim to have reduced their mortality to about $5 \%$. Butler ${ }^{3}$ and others ${ }^{4}$ have also reducad the mortality to such low level by essentially similar therapy except for the use of different electrolyte solutions, containing less potassium than was recommended by Darrow.

For many years we have employed ${ }^{5}$ systematic therapy for alimentary toxicosis. In view of the low mortality reported, we thought it advisable to examine our' results for the past 3 years and compare them with previous performances at the Mount Sinai Hospital of New York with the data mentioned. In reporting our findings we shall also dwell briefy on the concept of the disease which led to this therapeutic approach and on variols steps of the treatment.

There is fairly general agreement among pediatricians that the condition most commonly referred to as Alimentaty 
Toxicosis is in reality a sympton complex. It is a syndrome occurring in infants and young children resulting from a gastro-intestinal upset with diarrhea, regardless of its etiology. It is characterized by dehydration, blood concentration, acidosis, and in the severe cases, peripheral circulatory collapse and shock ${ }^{B}$ stressed by Powers. The reduced circulation results in diminished tissue oxygenation, and consequently there is metabolic disturbance both of anabolism and of catabolism. It is a general disturbance and involves the entire body causing dysfunction of the stomach, of the liver, of the pancreas, of the heart, of the brain and of the glandular systems both the endocrine and exoctine and especially of the kidney. It may be safe to assume that in the presence of reduced blood volume and reduced tissue oxygenation no part of the body is unaffected, not even the individual cell.

In the accompanying chart wich is self-explanatory, we have attempted to illustrate by diagram our concept of the various steps in the physiopathology of alimentary toxicosis. This concept is based on clinical and experimental observations made by many workers from the entire world.

This diagram is probably deficient since several features of the condition are not yet clear. Yet the physiology of the disease as described when considered along with the clinical appearance of the child with severe diarrhea and with previous experiences in the therapy of toxicosis, suggests a system of therapy a description of which follows:

\section{First day:}

1. Give nothing by mouth for twelve hours.

2. Start continuous intravenous drip of Ringer lactate solution plus $5 \%$ glucose. Administer about 35 cubic centimeters per kilogram of body weight in first $30 \mathrm{tg}$ 60 minutes, thereafter continue at approximately 150 cubic centimeters per kilogtam per 24 hours.

3. If hemoglobin is below 11 grams give transfusion, ap. proximately 20 cubic centimeters per kilogram (whole blood). If more than 11 grams give plasma, 25 to 35 cubic centimeters per kilogram. The plasma may be given before the initial injection of Ringer lactate solution.

4. When carbon dioxide content is known calculate requirement of $M / 6$ lactate necissary to increase the carbon 
.

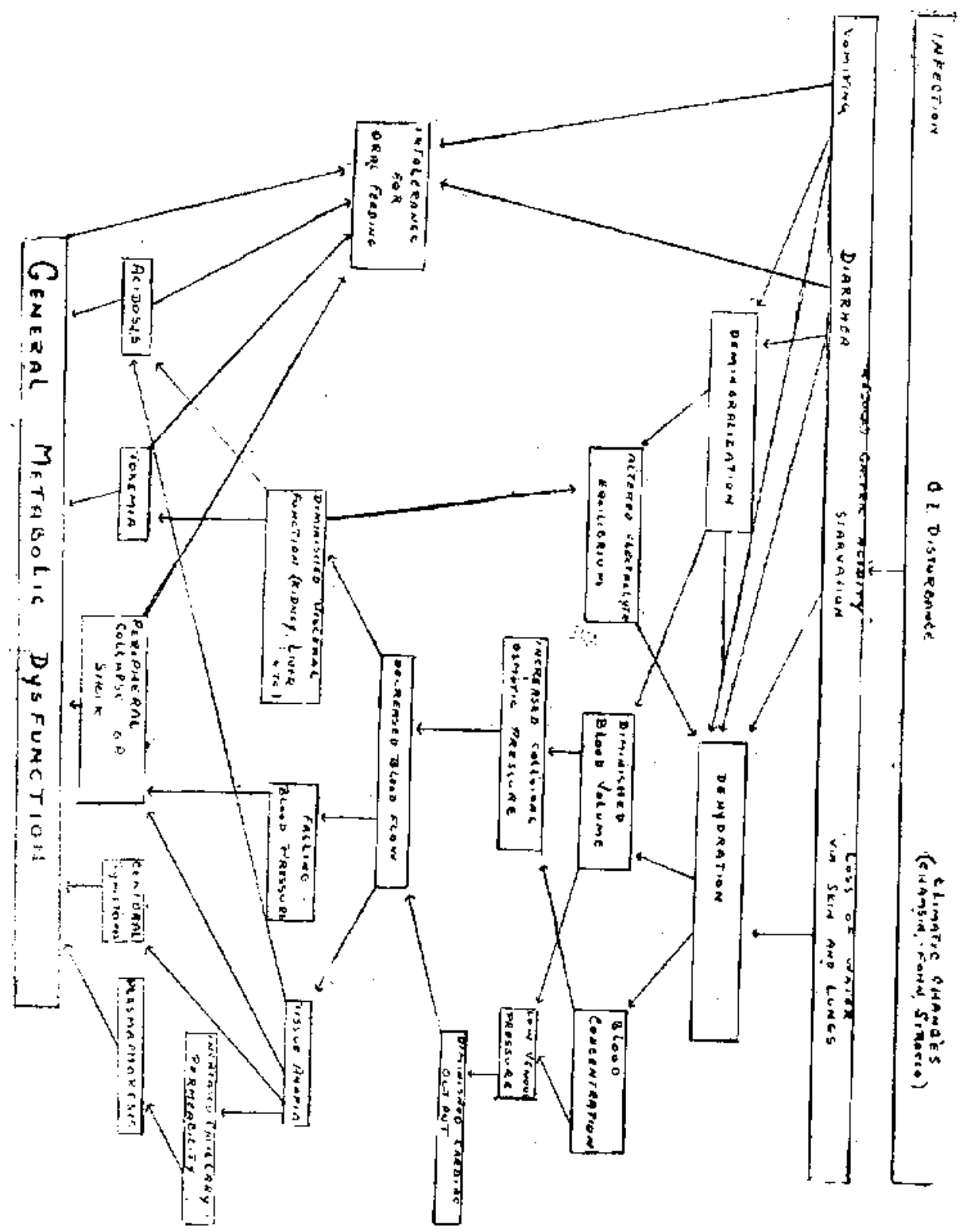


dioxide to $55 \mathrm{~V} / \%$ taking into account the lactate already injected. Give the calculated amount of $M / 0$ lactate with $5 \%$ glucose. Thereafter substitute balf strength physiologic saline or Ringer solution with $5 \%$ glucose. Darrow's solution may be used if diuresis has been established.

5. After the first twelve hours start water by mouth five or ten cubic centimeters every two hours, depending on the size of the child.

Second day and thereafter:

1. Continue the intravenous fluid, until $2 / 3$ of daily fluid quota is taken by mouth. Repeat blood or plasma as indicated. If the circulation is good and technical difficulties preclude the intravenous toute, administer the fluid not covered by mouth subcutaneously. It is preferable not to inject gluxcose subcutancously.

2. Increase water by mouth to 10 or 20 cubic centimeters every twro bours.

3. At the cnd of 36 hours if the child's condition is good. the diarrhea is improved, thete is no abdominal distention, and the child is thirsty, start formula of $2 / 3 \mathrm{milk}$. $1 / 3$ water, plus $5 \%$ sucrose, 10 calories per kilogram divided into 12 feedings, given every two hours. Lactic acid milk, skimmed lactic acid milk or butter-milk plus added carbchydrate may be substituted for above formula.

4. Make daily increases of formula at rate of 10 calories per kilogram until 40 or 50 calories per kilogram are given. Then the increase of 10 calories per kilogram may be made every second or third day. Water is increased daily by 5 or 10 cubic centimet? The water is usually given with the formula, except where the appetite is small; then give water after formula. Reduce number of feedings to 8 and later to 6 as is indicated by the child's appetite.

5. Discontinue parenteral fluid when requirements is satisfied by. oral feedings.

6. Abdominal distention and hypothermia usually imply difficulty. Distention indicates prompt measures for correction and reduction or elimination of oral feeding. 
Chart 2.

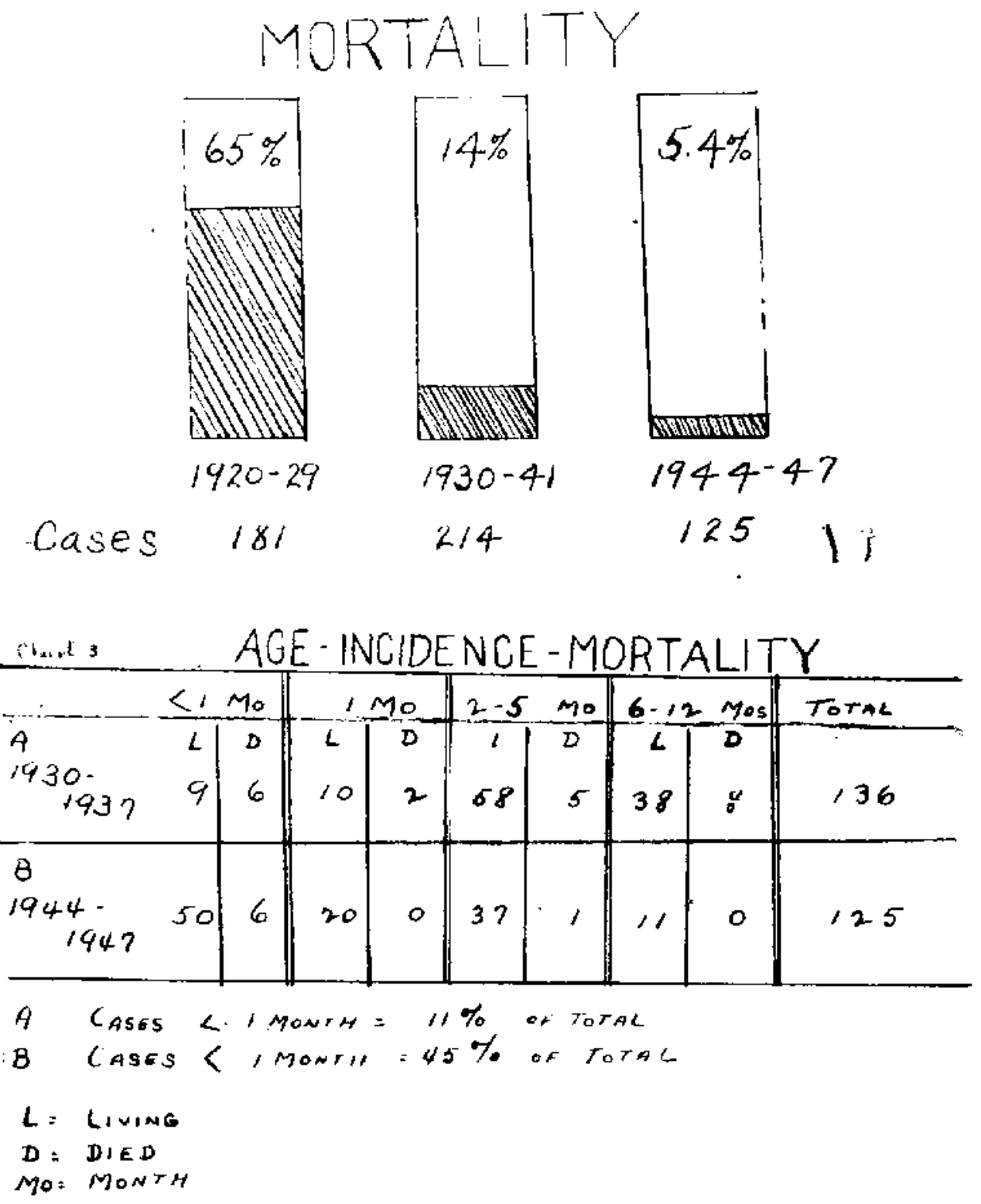


In case of recurrence of symptoms the entire program may have to be tepeated.

7. During the course of treatment serum protein, hematocrit, hemoglobin and red cell determination should be made frequently. Chemical determinations only as indicated.

8. If infection is present or suspected, penicillin or sulfadiazine may be included with the patenteral fluid. $\mathrm{Be}$ ginning the first day and there after vitamin $B$ and $C$ is to be supplied parenterally, daily until it can be given orally. A combination of thiamin and ascorbic acid may be used for this purpose. Amino acid preparations may be given intravenously if starvation is prolonged.

The system of therapy which we have employed consists of the intravenous administration of water, electrolytes, glucose, blood, plasma, sulfonamides or penicillin, vitamins and sometimes amino acids, a period of oral fasting and |realimentation by a slow staircase technic. The essential features of this treatment which has been used since 1929 aims at correction of what we consider the most important disturbances. These are: 1. Peripheral circulatory collapse. 2. Dehydration with its accompanying hemoconcentration, reduced bood volume and raduced blood flow. 3. Acidosis. 4. Impaired renal function. 5. Infection. 6. Diminished tolerance of food taken by mouth.

\section{Results of therapy.}

Chart 2 reveals that in the 10 years period from 1920 thru 1929, when no systematic therapy was employed the mortality was an average of $65 \%$. It ranged from $48 \%$ in the year of lowest mortality to $84 \%$ in the worst year.

From 1930 to 1941 the mortality was reduced to $14 \%$. The treatment during this period was essentially that describe in this report with the following exceptions. Isotonic sodium chloride solution with $5 \%$ added glucose or Ringer's solution with $5 \%$ added glucose were used for intravenous therapy almost exclusively except for the blowd and plasma transfusions and except for the periods of edema when these solutions were mixed with equal parts of $10 \%$ gluces in distilled water. Hartmans Ringer Lactate solution was eften substituted for the initial intravenous treatment after 1935 and occasionally before that. Vitamins were 
added to the intravenous therapy as soon as suitable thiamin chloride and ascorbic acid became available.

Sulfanilamide was introduced into the therapy in 1936 in form of prontosil and later was replaced by sulfadiazine. Penicillin had not yet become available during this period.

The data for our experience between 1941 and 1944 are now being reviewed, but cursory examination indicates a similar mortality as that in the period of 1930-1941.

The data of 1944-July 1947 reveal that in 125 cases triated with the routine as described the mortality was $5.4 \%$. If this period is broken down for each year, the results are as follows:

in 1944, of 26 cases, 3 died - a mortality of $13 \%$ in 1945, of 27 cases, 2 died - a mortaitity of $8 \%$ in 1946. of 49 cases, 1 died - a mortality of $2.1 \%$ and until July 1, 1947, of 28 cases, 1 died - a mortality of $3.6 \%$.

Thus, we too have observed a marked decline in the mortality of alimentary toxicosis in recent years. In the past 3 yrars it has been as low as that hitherto reported.

Assuming that our cases were of similar nature and of comparable intensity of illness as those in the various groups reported by Darrow, Butler, Wiehl et al. these results raise some pertinent questions. What are the factors which have reduced the mortality to this low level? How significant is intravenous therapy? Can this improvement be due to chemotherapy and antibiotics, or better treatment of the electrolyte deficiencies or to other factors? We shall attempt to answer some of these problens.

Continuous intravenous therapy.

It has been our feeling for a long time that the most important step in lowering the mortality is the initial phase of treatment, the correction of the collapsed or reduced circulation. If by fluid, whether it be physiologic sodium chloride solution plus glucose, Ringer's solution plus glucose or Hartman's or Butlers solution, the circulation is increased and as a result the blood concentration is lowered, renal function is increased and the acidosis is corrected then the patient is likely to recover. Experince indicates that this is pcissible in most cases with any of the solutions mentioned. 
We chose the continuous intravenous technic because after trying other methods of supplying fluid it was found to be the most suitable and most effective method for administering electrolyte solutions, blood or plasma, vitamins and sulfonamides or penicillin rapidly at first then slowly at at steady rate commensurate with the patients requirements and tolerance and with the least disturbance to the sick child. During the state of collapse fluids injected subcutaneously and intraperitoneally are not readily absorbed. Oral ferding in a child in stupor or one with severe nausea and diarrhea is defeated by vomiting, poor absorption and rapid excretion of the ingested fluids. Interrupted intravenous therapy is a much more difficult procedure for the average house officer and is quite exhausting for the infant pationt. Although the technic of continuous intravenous therapy is difficult it is not impossible, indeed it is or should be available in any hospita where childrs with aimentary toxicosis are treated. The condition is much too severe for treatment at home unless all necessary facilities are available. It is an emergency just as much as an acure surgical condition and should be treated with equal or greater concern. The duration of the continuous intravenous infusion must vary with the individual child and may be as short as 12 hours or extended over a pericid of many days. This depend on the ability of the infant to take adequate fluid by mouth and retain it.

Continuous intravenous therapy has been widely adopted for routine treatment of alimentary toxicosis as noted in the recent report of Wiehl, Dodd and Rappaport. In some clinics continuous intravenous thrapy is reserved for the treatment of the more severe cases. In these clinics milder cases have been treated with interrupted intravenous infusions, with subcutaneous injection and with oral feeding. We too have successfully treated cases of toxicosis of all severities without continuous intravenous therapy, but we have learned that it is not as regularly sucessful as is the routine including continuous intravenous therapy. It is often very difficult to properly evaluate the severity of a case of alimentaty toxicosis when it is first sien in the hospital. If errors in judgment are made one usually errs on the side of underestimating the degree of illness. Under such conditions a child is permitted to get worse by inadequate care. For this reason and because we have observed that in teaching internes better results are obtained by stressing uniformity of a the- 
rapeutic proceduce we prefat to start fluid administration by the continuous intravenous technic in all cases of alimentary toxicosis. Choice of therapy should be left to thos? who have experience and facilities, clinical and laboratory, for determining the most ideal approach for an individual child. Whire such facilities are missing and if the personnel are not experienced better results are to be had by observing a therapeutic routine such as is here described.

Effect of chemotherapy and antibiotics.

The introduction of sulfonamides and penicillin with the continuous intravenous therapy has been of great help and has undoubtedly increased the chances of survival. Pneumonia, otitis media, pycderma, pyelonephritis and other infections largely responsible for the death in many infants with dehydration, are now curid. Enteric infection due to many infants with dehydration, ate now cured. Enteric infection due to dysentery may respond to sulfonamides. Cooper ${ }^{8}$ et al. of the United States and more recently L. F. Meyer $^{3}$ and $A$. $A$. Aballi ${ }^{\text {to }}$ have indicated the beneficial effect of sulfathiazol in treatment of toxicosis in Palestine and Cuba in cases infected with dysentery.

Choice of fluid for intravenous therapy.

Ringer Lactate solution has been substituted for the Ringer's solution or physiologic sodium chloride solution in the initial phase of treatment because the acidosis is thus ccrrected more rapidly and because edima develops less often. When larger amounts of sodium chloride were injected edema developed in most children and the acidosis was more difficult to overcome.

To date we have used Darrow's solution only after the circulation and renal function is improved. or after survival of the child seems reasonably assured. Our experience is too meager to justify comment other than that we have observed no ill effect from its use and that it is hoped that by the more frequent use of potassium there will be a reduction in the incidence of secondary breakdown which is still a frequent accurrence.

Blood plasma and whole blood transfusions have been used more freely in recent years and it is onr belief that this has helped to maintain better circulation. Many cases have 
been successfully treated without either blood or plasma but our experience indicates that sicondary breakdown is less common after use of blood or plasma.

Period of eral starvation and choice of formpla.

From time to time members of cur staff have attempted to shorten the period of oral starvation from the minimum of 36 hours as recommended. While it has bien successful in some it has also been the cause of secondary breakdown. We believte therefore that this period of oral starvation should be for the minimum pericd of 36 hours except for water. In our hospital some have preferred protein milk, others skimmed lactic acid milk, still other human breast milk instead of a simple diluted sweet milk as suggested in the outline of treatment. The low hydrochloric acid content of gastric juic of such infants does indicate a possible advantage for acidified milk. Yet there seemed to be no correlation between the type of formula first offered and the recovery except in a small percent of the cases. Except in the case of food allergy or of coeliac disease and of few others the quantity of formula first given rather than its type seemed to have been most important. Butler has recently come to this same conclusion.

\section{Epidemic diarrhea of the newborn.}

For several years here has been a widespread occurrence of a very violent epidemic infection in newborn infants cansing diarrbea and rapid dehydration and collapse with a mortality of $40-50 \%$. It is called epidemic diarrhea of the newborn. While most epidemics were very severe, others were associated with little or no mortality. In the last few years some of the patients sent to us diagnosed epidemic diarrbea of the newbotn have been in better condition on arrival or if in severe acidosis and collapse responded to treatment more readily. It was surprising that they responded to the exact same therapy which had failed to help a high percent of the cases treated.

These observations suggest siveral possibilities, first that due to a better appreciation of the disease we ate starting treatment earlier, second that antibiotics and chemotherapy are belpful either against the actual etiologic agent or against complicating bacteria and third and possibly most important 
is the likelihood that in most outbreaks the disease is different and less virulent than it was. These patients are admitted thruout the year. Many of them occur sporadically rather than in epidemics. For such cases as occur sporadically the name Diarrbea of the Niwborn rather than Epidemic Diarthea of the Nifwborn seems preferable.

S:multaneous with the increase of toxicosis in newborn infants we have observed a decline in incidence of the disease in children 6 menths of age or older in the vicinity of Mount Sinai Hospital. This wa belicve to be associated with the more general use of pasteurized milk or evaporatad milk and due to the better control of parenteral infection with chemotherapy and antibiotics, and to gr:ater dissemination of information among parents about the significance of diarrhea in babies and to better education of doctors and ail child caring personnel in the treatment of simple diarthea.

\section{In conclusion.}

We are reporting a mortality of $5.4 \%$ in the treatment of alimentary toxicosis in 125 cases treated between 1944 and 1947. This compares with a mortality of $14 \%$ after the introduction of systematic therapy with continuous intravenous administration of fluid and a mortality of $65 \%$ before that period.

From the good results reported by various workers, it would seem that it is the combination of the therapeutic measures recommended which has brought about these results. Indeed other factors not controlled by us, such as altered virulence of infection in the newborn may also have influenced the results.

The therapeutic procedure cutlined can be employed quite generally and modified for such conditions as prevail in different localities. While best results are to be expectex! by careful laboratory control and adjusting therapy in acccrdance with the findings, it is possible to carry out this procedure as indicated in the outline of treatment with a minimum of laboratory procedure. 


\section{Bibliography.}

I. DARROW, D, C, - J. Pediat, 28: 515, 1946.

2. GOVEN, C. D. J. and DARROW. D. C. - J. Pediat. 28: $541,1946$.

3. BUTLER. A. N.: REYERSBACH, G. C. and TALBOT, N. B. - - Read before The American Pediattic Society. May 14, 1947.

4. WEIHL. M. D.; RAPOFORT. S. and DODD, K. -- J. Pediat. 30 : 45. 1947 .

5. KARELITZ, S. and SCHICK, B. - Am. J. Dis. Cbild, 42; 781. 1931 .

6. POWERS, G. G. - Am. J, Dis, Child. 32: 232, 1926.

7. BUTLER, A. N. MCKHANN. C. F. and GAMBLE, J. L. - J. Pediat. 3: 84, 1933 .

8. COOPER, M. L.; ZUCKER, R. L. and WMGONER, S. - J. A. M. A. 117: 1520, 1941.

9. MEYER, L. F. 一 Read before the 5 th International Pediatries Congress. New York, Jaly 18, 1947.

10. ABAI.LI, A. A. - Idem. 